Apidologie, 1982, 13 (3), 275-295.

\title{
WITTERUNGSVERLAUF UND MASSENWECHSEL DER GRÜNEN TANNENHONIGLAUS CINARA PECTINATAE (NÖRDL.) (HOMOPTERA, LACHNIDAE) IN DEN JAHREN 1977-1981
}

\author{
Gerhard LIEBIG (1), Ursula SCHLIPF (1) und Waltraud DÜWEL (2)
}

\section{ZUSAMMENFASSUNG}

Von 1977 bis 1981 wurde in Hohenheim an sechs 15- bis 20-jährigen Tannen ( $A$ bies alba) der Massenwechsel von Cinara pectinatae mit dem Witterungsverlauf verglichen. Die Populationsdichte wurde von April bis Oktober in 1- bis 2-wöchigen Abständen durch Auszählen der Lachniden auf markierten Zweigen (etwa $20 \times 30 \mathrm{~cm}$ gross, je Baum 10) festgestellt. Der Witterungsverlauf wurde dargestellt mit den Tagesund Monatsmitteln der Lufttemperatur sowie mit den Niederschlägen als Tagesmengen und als monatliche Gesamtsummen.

Der Massenwechsel von C. pectinatae läuft jedes Jahr nach einem Schema ab, das sich von März bis Oktober in 4 bzw. 5 Phasen einteilen lässt : 1. Entwicklungszeit der Stammütter (März bis Mai), 2. Vermehrungsphase (Mai/Juni), (3.) Stagnationsphase (Juni), 4. (oder 3.) Zusammenbruch (Juli/August), 5. (oder 4.) Auftreten der Geschlechtstiere (September/Oktober).

Eine deutliche Vermehrung der Lachniden findet in der Regel nur während des Austriebes im Mai und Juni statt. 1978 und 1980 wurde im Juni mit durchschnittlich 80 bzw. 90 Lachniden je 10 Triebe ein Befall festgestellt, der 2- bis 3-mal so hoch war wie 1977, 1979 und 1981. C. pectinatae hatte sich 1978 und 1980 stärker vermehrt als 1977, 1979 und 1981, obwohl Mai und Juni kälter und 1978 auch niederschlagsreicher waren. Der direkte Vergleich von Massenwechsel und Witterungsverlauf zeigt, dass die Veränderungen in der Populationsdichte nicht durch Temperaturschwankungen und Niederschläge verursacht werden.

Fruchtbarkeit und Sterblichkeit der Lachniden werden wahrscheinlich in erster Linie durch den physiologischen Zustand des Wirtsbaumes beeinflusst. Die Untersuchungen werden mit der Arbeitshypothese fortgesetzt, dass die Unterschiede im Massenwechsel zwischen den Jahren bzw. zwischen Standorten auf eine witterungs- und standortbedingte unterschiedliche Nährstoffversorgung der Weisstannen zurückzuführen sind.

(1) Landesanstalt für Bienenkunde der Universität Hohenheim, Postfach 700562 , D-7000 Stuttgart 70.

(2) Institut für Physik der Universität Hohenheim. 


\section{EINLEITUNG}

Die Grüne Tannenhoniglaus Cinara pectinatae ist der bedeutendste Honigtauerzeuger auf der Weisstanne Abies alba. Zu einer Tannentracht im Juli/Augusi kommt es nur dann, wenn eine intensive Vermehrung der Lachniden im Mai, Juni und Juli stattfindet und zu einem Massenbefall mit entsprechend starken Honigtauausscheidungen führt.

Nach der sogenannten Gradozöntheorie, wie sie SCHWERdTFEgER (1944) darstellte, ist der Massenwechsel von einer Vielzahl von Faktoren abhängig. Es sind dies in der Hauptsache Witterungs- und Standortfaktoren, das Nahrungsangebot, Parasiten, Räuber und Krankheiten und die Art selbst mit ihren gradologischen Eigenschaften. Komplex ist der Einfluss der Witterung auf den Massenwechsel der Lachniden; sie kann direkt auf die Lachniden wirken oder auch indirekt über die Wirtspflanze, über den Standort und über Feinde und Krankheitserreger.

Von verschiedenen Trachtbeobachtern wurde das Auftreten von sogenannten Fehljahren wiederholt damit erklärt, dass eine ungünstige Witterung im Frühjahr und Frühsommer die Massenvermehrung der Honigtauerzeuger verhindert hätte. LEONHARDT (1940), Wille (1966, 1967), WelleNSTEIN (1977) und Wütherich (1977) untersuchten den Witterungscharakter von Waldhonigjahren und Fehljahren, indem sie den Honigertrag mit dem Witterungsverlauf verglichen. Während nach WILLE keine eindeutigen Beziehungen zwischen der Witterung und der Waldtracht bestehen, fanden LeONHARDT, WellensteIN und Wütherich, dass in guten Waldhonigjahren Frühjahr und Sommer überdurchschnittlich warm sind, in Fehljahren dagegen $\mathrm{zu}$ feucht und zu kalt. Sie folgerten daraus, dass die Entwicklung eines Massenbefalls der Lachniden durch eine trockene und warme Witterung begünstigt wird.

Der Honigertrag allein scheint nicht geeignet, den Massenwechsel von Lachniden zu beurteilen, weil er nicht nur von der Befallsdichte der Honigtauerzeuger abhängt, sondern auch von der Leistungsstärke der Bienenvölker, von den Flugbedingungen während einer Honigtautracht und von der Attraktivität des Honigtaus. Ausserdem wurde bei dem Vergleich des Honigertrages mit der Witterung nicht berücksichtigt, dass die verschiedenen Honigtauerzeuger, die an einer Waldtracht beteiligt sein können, aufgrund ihrer gradologischen Eigenschaften unterschiedlich stark witterungsempfindlich sind.

Die Zusammenhänge zwischen dem Witterungsverlauf und dem Zustandekommen einer Waldtracht dürften sich daher klarer herausarbeiten lassen, wenn man sich zunächst auf den Vergleich zwischen dem Massenwechsel der Honigtauerzeuger und den meteorologischen Daten beschränkt. Dies geschieht in der vorliegenden Arbeit mit dem Massenwechsel von C. pectinatae am Standort Hohenheim für die Jahre 1977-1981. 


\section{METHODIK UND MATERIAL}

\section{Beobachtung des Massenwechsels 1977-1981}

Vor dem Gebäude der Landesanstalt für Bienenkunde in Stuttgart-Hohenheim steht eine Anpflanzung von etwa je 40 Tannen und Fichten im Alter von 15-20 Jahren. Jedes Jahr im März wurden an 6 Tannen (1977 an 3 Tannen) je 10 Triebe markiert. Jeder Trieb war mit seinen Verzweigungen etwa $20 \mathrm{~cm}$ breit und etwa $30 \mathrm{~cm}$ lang; die ausgewählten Triebe befanden sich sowohl an der Peripherie als auch im Inneren des Baumes. Die Triebe waren am Baum rundum in Hüft- bis Augenhöhe verteilt. Je nach ihrer Stellung am Baum bestanden die Triebe aus dem Zuwachs von 2 bis 5 Jahren.

1977,1978 und 1979 wurde der Massenwechsel von C. pectinatae an anderen Tannen beobachtet als 1980 und 1981.

Von Mai bis Oktober wurde der Lachnidenbesatz auf den markierten Trieben in wöchentlichen Abständen gezählt. Die Anzahl der auf 10 Trieben einer Tanne sitzenden Lachniden wurde addiert, von dem Lausbesatz der 6 bzw. 3 Tannen (1977) wurde der Mittelwert gebildet.

C. pectinatae tritt auch bei Massenbefall als Distanztier (Einzelsitzer) auf. Neugeborene Larven sind etwa $1 \mathrm{~mm}$ gross und blassgrün gefärbt; erwachsene Tiere sind zwischen 4 und $6 \mathrm{~mm}$ gross, nur selten geflügelt, dunkelgrün mit weissen Rückenstreifen und einer matt silbern glänzenden Bauchseite. Biologie und Entwicklungszyklus von $C$. pectinatae wurden wiederholt beschrieben (z. B. KLOFT et al., 1965; Maquelin, 1974; Liebig, 1979 b).

Bei den Zählungen wurden die markierten Triebe sorgfältig auf der Ober- und Unterseite abgesucht. Bei einiger Übung sind die zwischen den Tannennadeln an der Rinde saugenden Lachniden sicher zu erkennen. Zur gleichen Zeit von 2 Personen unabhängig voneinander durchgeführte Zählungen brachten gleiche oder nur geringfügig abweichende Resultate.

Neben den Messungen der Populationsdichte wurde der Schlüpftermin der Stammutterlarven, das Auftreten der ersten $F_{1}$ und der Beginn der Eiablage (nur 1977, 1978 und 1981) festgehalten. Der Schlüpftermin wurde durch laufende Kontrollen markierter Eier ab Ende Februar ermittelt.

2. Zusammenstellung der Witterungsdaten

Für den Vergleich des Massenwechsels mit dem Witterungsverlauf wurden die Klimadaten der Wetterstation Hohenheim verwandt, die ca. $600 \mathrm{~m}$ nördlich der Landesanstalt liegt. Ausgewertet wurden Tages- und Monatsmittel der Lufttemperatur sowie die Niederschläge als Tagesmengen und als monatliche Gesamtsummen.

\section{ERGEBNISSE}

\section{Allgemeines zum Massenwechsel von C. pectinatae}

Der Massenwechsel von C. pectinatae läuft jedes Jahr nach einem Schema ab, das sich von März bis Oktober in 4 bzw. 5 Phasen einteilen lässt.

\section{Entwicklungszeit der Stammütter}

Sie beginnt mit dem Schlüpftermin der Stammutterlarven und endet mit dem Auftreten der ersten $F_{1}$.

1977 schlüpften die Stammütter Anfang März aus den überwinterten Eiern, 1978 Ende März, 1979 und 1980 erst Anfang April und 1981 Mitte März (vgl. Tab. 1). 
TAB. 1. - Zeitliche Daten des Massenwechsels von C. pectinatae 1977-1981.

TABL. 1. - Important times of the population trend of C. pectinatae 1977-198I.

\begin{tabular}{|c|c|c|c|c|c|}
\hline & 1977 & 1978 & 1979 & 1980 & 1981 \\
\hline $\begin{array}{l}\text { Schlüpfzeit der } \\
\text { Stammutterlarven }\end{array}$ & $\begin{array}{l}\text { Anfang } \\
\text { März }\end{array}$ & $\begin{array}{l}\text { Ende } \\
\text { März }\end{array}$ & $\begin{array}{c}\text { Anfang } \\
\text { April }\end{array}$ & $\begin{array}{l}\text { Anfang } \\
\text { April }\end{array}$ & $\begin{array}{l}\text { Mitte } \\
\text { März }\end{array}$ \\
\hline Hatching of the fundatrice larvae & $\begin{array}{l}\text { Early } \\
\text { March }\end{array}$ & $\begin{array}{l}\text { Late } \\
\text { March }\end{array}$ & $\begin{array}{l}\text { Early } \\
\text { April }\end{array}$ & $\begin{array}{l}\text { Early } \\
\text { April }\end{array}$ & $\begin{array}{l}\text { Middle } \\
\text { of March }\end{array}$ \\
\hline $\begin{array}{l}\text { Auftreten der ersten } \mathbf{F}_{1}(=\text { Beginn } \\
\text { der Vermehrungsphase) }\end{array}$ & $\begin{array}{l}\text { vor dem } \\
\text { before }\end{array}$ & & & & \\
\hline $\begin{array}{l}\text { Appearance of the first } F_{1}(=\text { beginning } \\
\text { of the reproductive phase) }\end{array}$ & 9. 5 . & 9. 5. & 21. 5 . & 21. & 28. 4. \\
\hline $\begin{array}{l}\text { Ende der Vermehrungsphase } \\
\text { End of the reproductive phase }\end{array}$ & 23. 5 . & 27. 6. & 12. 6. & 26. 6. & 11. 6. \\
\hline $\begin{array}{l}\text { Beginn des Zusammenbruchs } \\
\text { Beginning of the decrease }\end{array}$ & 21. 6. & 27. 6. & 27. 6. & 26. 6. & 26. 6. \\
\hline $\begin{array}{l}\text { Ende des Zusammenbruchs } \\
\text { End of the decrease }\end{array}$ & 29. 7. & 21. 8 . & 13. 8. & 2. 9. & 3. 8. \\
\hline $\begin{array}{l}\text { Beginn der Eiablage } \\
\text { Start of oviposition }\end{array}$ & 14. 10 . & 17. 10 . & \multicolumn{2}{|c|}{$\begin{array}{l}\text { nicht festgestellt } \\
\text { not ascertained }\end{array}$} & 30. 9. \\
\hline
\end{tabular}

Die Stammutterlarven benötigten für ihre Entwicklung zum erwachsenen, vermehrungsfähigen Tier 6-8 Wochen. Die ersten $F_{1}$ wurden 1981 am 28.4., 1980 am 21.5., 1979 am 21.5. und 1978 am 9.5. gefunden. 1977 wurde erst am 9.5. mit den Zählungen begonnen, als neben den erwachsenen Stammüttern schon $F_{1}$ vorhanden waren.

2. Vermehrungsphase

Mit dem Beginn der Larvenproduktion durch die Stammütter steigt die Populationsdichte ständig an. 1977 wurde nur bis zum 23.5. eine signifikante Zunahme des Lachnidenbesatzes festgestellt. 1979 und 1981 dauerte die Vermehrungsphase bis zum 12.6. bzw. 11.6., 1978 und 1980 dagegen bis zum 27.6. bzw. 26.6. (vgl. Abb. 1, 2, 4,5 und 7).

\section{Stagnationsphase}

Nach der Yermehrungsphase bleibt der Lachnidenbesatz für bis zu 4 Wochen relativ konstant. Dies scheint allerdings nur dann der Fall zu sein, wenn die Vermèhrungsrate relativ niedrig liegt wie 1977, 1979 und 1981 bzw. 1980 auf Baum 4 (vgl. Abb. 6).

\section{4. (oder 3.) Zusammenbruch}

Im Juli und August nimmt der Lachnidenbesatz regelmässig ab. Nach einem starken Befall erfolgt der Zusammenbruch im Anschluss an die Vermehrungsphase und rascher als nach einem schwachen Befall. 
5. (oder 4.) Auftreten der Geschlechtstiere

Im September stabilisiert sich der Lachnidenbesatz mit dem Auftreten der Geschlechtstiere auf einem relativ niedrigen Niveau. Dabei kann es wie 1977 und 1981 zur Ausbildung eines zweiten, aber sehr niedrigen Populationsgipfels kommen. Die ersten Geschlechtstiere sind Anfang Oktober erwachsen. Es kommt zur Kopulation und anschliessend zur Eiablage durch die Weibchen.

2. Der Vergleich des Massenwechsels mit dem Temperaturverlauf und den Niederschlägen

1977 (Abb. 1) : 1977 wurde erst am 9.5 mit den regelmässigen Zählungen begonnen. Im Durchschnitt wurden 6 Tiere je Baum gezählt, 3 erwachsene Stammütter

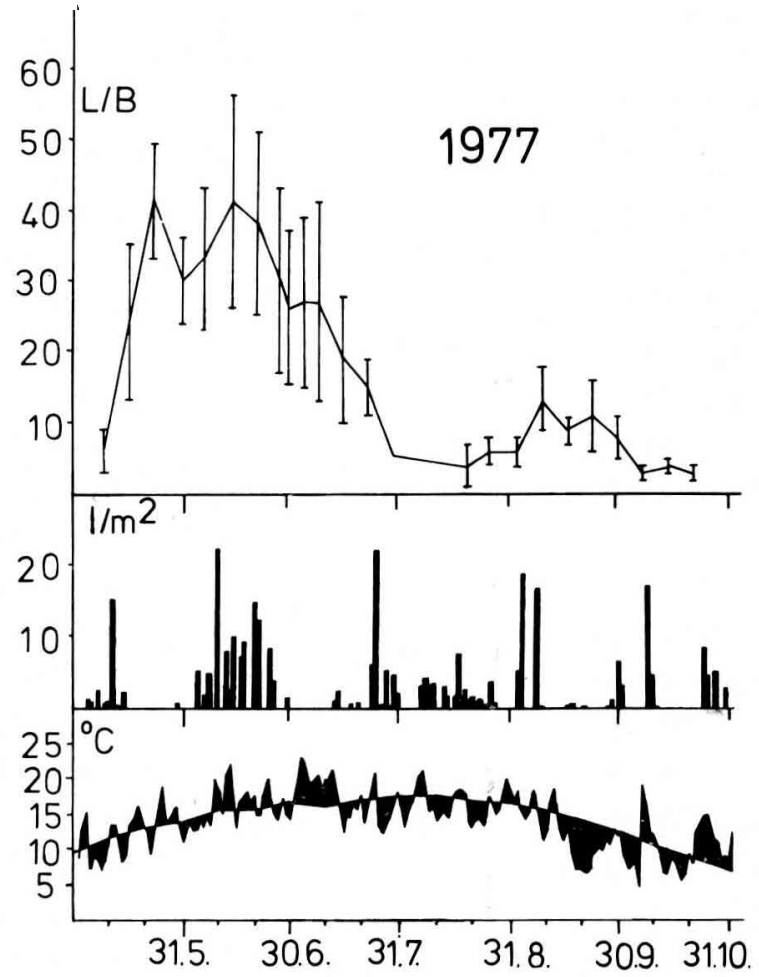

Aвв. 1. - Der Massenwechsel von C. pectinatae und der Verlauf der Witterung 1977 von Mai bis Oktober.

L/B = durchschnittlicher Lachnidenbesatz je Baum, aufgezeichnet sind die Mittelwerte und die Standardabweichungen.

$1 / \mathrm{m}^{2}=$ tägliche Niederschlagsmenge in Liter je $\mathrm{m}^{2}$.

${ }^{\circ} \mathrm{C}=$ Tagesmittel der Temperatur; die Abweichungen vom langjährigen Mittel sind durch die dunklen Flächen verdeutlicht.

FIG. 1. - Population trend of $C$. pectinatae $(L / B)$ with temperature $\left({ }^{\circ} C\right)$ and rainfall data $\left(1 / \mathrm{m}^{2}\right)$ in 1977 .

$\mathrm{L} / \mathrm{B}=$ population means on each tree and standard deviations.

$1 / \mathrm{m}^{2}=$ daily rainfall in litre $/ \mathrm{m}^{2}$.

${ }^{\circ} \mathrm{C}=$ daily mean of temperature deviations from long standing means are drawn as dark areas. 
und $3 F_{1}$. Die Populationsdichte stieg bis zum 23.5. auf durchschnittlich 41 Tiere je Baum an. Bis zum 31.5. sank der Besatz um ca. $25 \%$ auf 30 Tiere ab, um anschliessend in der ersten Junihälfte erneut auf 41 Tiere je Baum (am 14.6.) anzuwachsen. Danach war ein langsamer und ständiger Rückgang in der Populationsdichte zu beobachten. Am 29.7. wurde ein Minimum von 6 Tieren je Baum gezählt.

Die Witterung im Mai, Juni und Juli 1977 war geprägt durch die Aufeinanderfolge von langandauernden Regen- und Trockenperioden. Vom 5.5. bis zum 15.5. fielen an 7 Tagen Niederschläge, die jedoch mit Ausnahme derjenigen vom 12.5., als $15,11 / \mathrm{m}^{2}$ gemessen wurden, minimal blieben. Vom 16.5. bis zum 4.6. fehlte nahezu jegliche nennenswerte Niederschlagstätigkeit, bis es ab 5.6. bis zum 26.6. zu häufigen und mehrmals kräftigen Niederschlägen kam. Während dieses Zeitraumes kam es zu 16 Tagen mit Regen, an 9 Tagen davon wurden mehr als $5 \mathrm{l} / \mathrm{m}^{2}$ festgestellt. Dem übernormal nassen Juni folgte der trockene Juli; erst während der letzten 8 Monatstage fielen Niederschläge in recht unterschiedlicher Stärke.

Der Temperaturverlauf war im Mai, Juni und Juli insgesamt ausgeglichen. Im Mai und Juni wechselten sich wärmere und kältere Tage häufig ab, der Juli dagegen war gekennzeichnet durch eine hochsommerliche und sehr sonnige erste Monatshälfte, der überwiegend zu kühle, wolkenreiche und damit zu trübe Witterung bis Monatsende folgte.

Während der Vermehrungsphase 1977, die bis zum 23.5. dauerte, fiel nur am 12.5. starker Niederschlag. Die Vermehrungsphase ging in der Trockenperiode dieses Monats (die sich über die letzte Dekade erstreckte) zu Ende, anschliessend war bei anhaltend trockener Witterung ein kurzfristiger Rückgang in der Populationsdichte zu beobachten. In der ersten Junihälfte stieg der Lachnidenbesatz noch einmal bei niederschlagsreicher Witterung an. Am 10.6. wurde bei Gewitter etwa während 10 Minuten Hagelfall beobachtet, die Niederschlagsmenge betrug an diesem Tag $22,2 \mathrm{l} / \mathrm{m}^{2}$, was sich offensichtlich nicht auf den Lachnidenbesatz auswirkte.

Der nach dem 15.6. einsetzende Zusammenbruch begann während der Niederschlagsperiode in der zweiten Junihälfte, setzte sich fort unter der hochsommerlichen, trockenen Witterung in der ersten Junihälfte und war beendet nach den gewittrigen, starken Regenfällen in der letzten Dekade des Juli. Es ist insgesamt nicht zu erkennen, dass der Zusammenbruch durch Niederschläge beschleunigt bzw. durch trocken-warme Witterung gebremst wurde.

Im August, September und Oktober wurden nur geringfügige Schwankungen in der Populationsdichte festgestellt. Vom 2.9. bis zum 9.9. verdoppelte sich der Besatz von 6 auf 12 Tiere je Baum, obwohl in diesem Zeitraum am 4. und am 8.9. starke Niederschläge fielen $\left(18,9\right.$ und $\left.16,7 \mathrm{l} / \mathrm{m}^{2}\right)$. Der Lachnidenbesatz ging dann bei überwiegend trockener und kalter Witterung auf 3 Tiere je Baum im Oktober zurück. Am 14.10. wurden auf den markierten Zweigen die ersten Eier gefunden. 
1978 (Abb. 2 und 3) : Vom 3.4. bis zum 16.5. wurden bei insgenamt 6 Zählungen jedesmal durchschnittlich 2 Stammütter je Baum gezählt. Während der Entwicklungszeit der Stammütter war das Wetter bis zum 17.4. wechselhaft, insgesamt zu kalt mit schauerartigen Niederschlägen, die als Regen-, Schnee- oder Graupelgemisch fielen. Vom 21.4. bis zum 5.5. herrschte leicht unbeständige, aber milde Witterung. Die sich anschliessende Abkühlung hielt bis zum 15. Mai an, wobei es lediglich am 12.5. zu geringem Bodenfrost kam.

Nach dem 16.5. begann die Vermehrungsphase. Bis Ende Juni stieg die Populationsdichte im Mittel der 6 Bäume ständig an. Sie erreichte am 28.6. mit durchschnittlich 84 Tieren je Baum das Jahresmaximum.

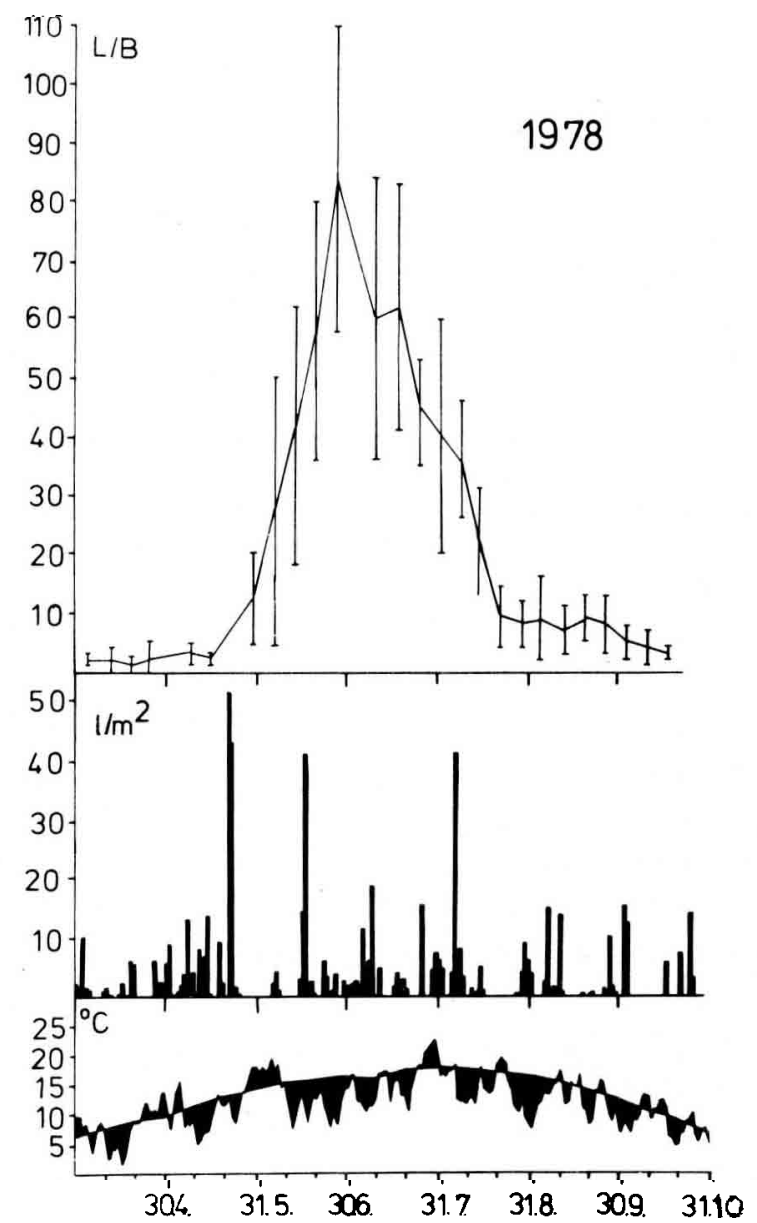

Aвв. 2. - Der Massenwechsel von C. pectinatae und der Verlauf der Witterung 1978 von April bis Oktober.

Populationsdichte, Nierderschlagsmenge und Temperatur wie in Abb. 1.

FIG. 2. - Population trend of $\mathrm{C}$. pectinatae with temperature and rainfall data in 1978 (from April to October).

Population density, rainfall and temperature as in Fig. 1. 


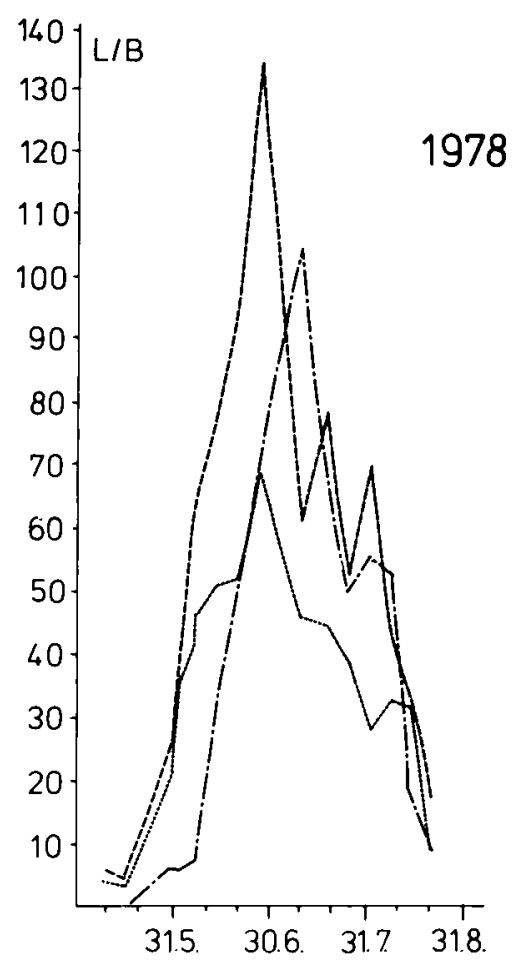

ABB. 3. - Der Massenwechsel von C. pectinatae 1978 von Mai bis August auf Baum 1 (..-……..),

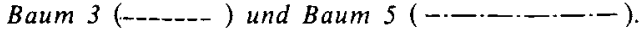

Fig. 3. - Population trend of C. pectinatae in 1978 (May to August)

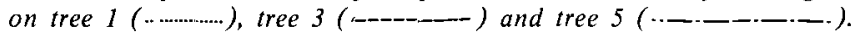

Während der Vermehrungsphase trat am 22.5. ein Gewitter auf, das die für rund 40 Stunden lang anhaltenden, wolkenbruchartigen Niederschläge einleitete. Vom 16.5 . bis zum 30.5 stieg die Populationsdichte von 2 auf 12 Tiere je Baum an. Anschliessend nahm der Lachnidenbesatz bis zum 27.6. im Durchschnitt nahezu linear zu; das war sowohl während der sommerlichen Periode vom 28.5. bis zum 9.6. der Fall als auch während der kühlen und niederschlagsreichen zweiten Monatshälfte. Gewittrige Starkniederschläge am 16. und 17.6. mit über $501 / \mathrm{m}^{2}$ Regen zeigten keine Auswirkungen auf den Populationsanstieg. Ein Einfluss der Witterung ist auch dann nicht zu erkennen, wenn man den Massenwechsel auf einzelnen Bäumen miteinander vergleicht (Abb. 3). Der Anstieg der Populationsdichte verlief auf Baum 1 und 3 vom 16.5. bis zum 2.6. nahezu gleich (auf 36 Lachniden je 10 Triebe auf Baum 1, auf 38 Lachniden je 10 Triebe auf Baum 3). Danach verdoppelte sich die Populationsdichte auf Baum 1 bis zum 27.6. auf 69 Tiere, auf Baum 3 dagegen stieg sie um das Vierfache auf 133 Tiere je 10 Triebe an.

Auf Baum 1 und 3 wurde der stärkste Befall am 27.6. festgestellt, auf Baum 5 dagegen erst am 10. Juli. 
Diese Unterschiede zwischen den 3 Bäumen zeigen, dass die Witterung weder die Dauer der Vermehrungsphase noch die Intensität der Vermehrungstätigkeit direkt beeinflusst.

Das gilt auch für den Zusammenbruch. Der Befall nahm 1978 im Durchschnitt im Juli und August ständig ab, was sowohl bei kalt-trockener Witterung als auch bei warm-feuchter und kalt-feuchter Witterung der Fall war. Die stärksten Niederschläge in der Phase des Zusammenbruchs fielen am 9.7. mit $18,8 \mathrm{l} / \mathrm{m}^{2}$, am 26.7 . mit $15 \mathrm{l} / \mathrm{m}^{2}$ und am 7.8. mit $41,31 / \mathrm{m}^{2}$. Durch diese Regenfälle wurde der Zusammenbruch nicht beschleunigt.

Lediglich vom 10.7. bis zum 18.7. wurde ein einmaliger leichter Anstieg von durchschnittlich 60 auf 62 Tiere je Baum festgestellt. In dieser Zeit war es relativ trocken bei durchschnittlich etwas unternormalen Temperaturen. Während einer vergleichbaren Wetterlage zwischen dem 14.8. und 21.8. nahm der Befall dagegen deutlich von 22 auf 9 Tiere je Baum ab.

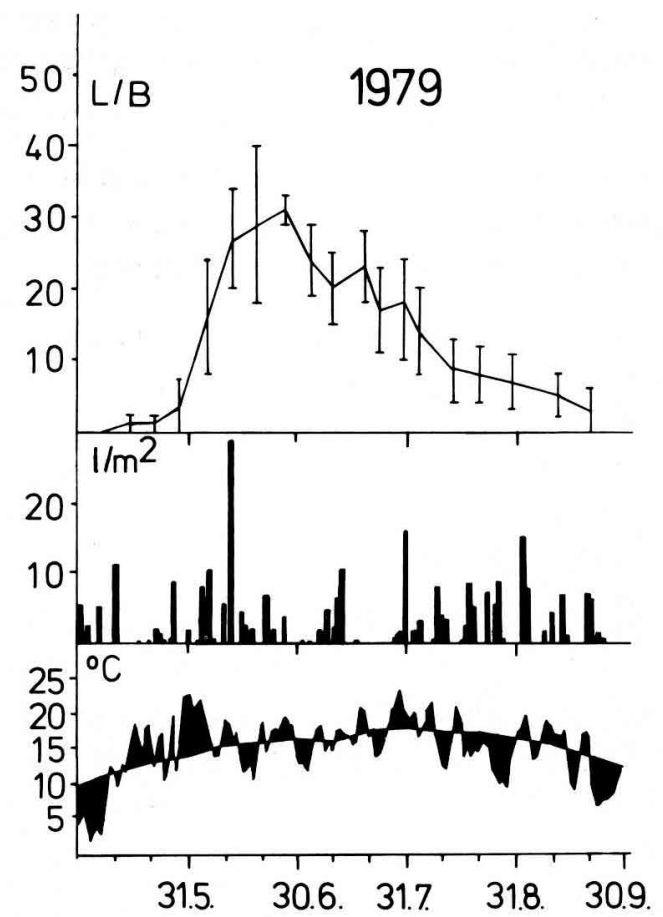

Aвв. 4. - Der Massenwechsel von C. pectinatae und der Verlauf der Witterung 1979 von Mai bis September.

Populationsdichte, Niederschlagsmenge und Temperatur wie in Abb. 1.

FIG. 4. - Population trend of $\mathrm{C}$. pectinatae with temperature and rainfall data in 1979 (May to September).

Population density, rainfall data and temperature as in Fig. 1. 
Von Ende August bis Anfang Oktober blieb die Populationsdichte relativ konstant mit leicht abnehmender Tendenz. Die leichten Schwankungen stehen in keiner Beziehung zu dem Witterungsverlauf im September und Oktober.

1979 (Abb. 4) : 1979 wurde im Mai durchschnittlich eine Stammutter je Baum gezählt. Die Vermehrungsphase setzte zwischen dem 21.5. und dem 28.5. ein. Besonders stark war der Anstieg in der Populationsdichte vom 28.5 bis zum 12. Juni. Nach dem 12.6. war nur noch eine schwache, nicht signifikante Zunahme zu beobachten. Eine deutliche Vermehrung fand somit - wie 1977 - nur während eines Zeitraumes von etwa 14 Tagen statt. Anschliessend blieb der Lachnidenbesatz ca. 4 Wochen lang (bis zum 9.7.) relativ konstant, es wurde insgesamt nur eine leichte Abnahme registriert.

Wie 1977 ist zu erkennen, dass die verglichen mit 1978 kürzere Vermehrungsphase nicht durch einen direkten Einfluss der Witterung verursacht wird. Das Abflachen des Anstiegs in der Populationsdichte nach dem 12.6. erfolgte bei einer Witterung mit insgesamt durchschnittlichen Temperaturen und - verglichen mit der Zeit der stärksten Vermehrung - geringen Niederschlägen. Am 12.6. wurde der Lachnidenbesatz vor und nach einem gewittrigen Starkniederschlag (mit etwa 5 Minuten lang anhaltendem schädigendem Hagel) gezählt. Weder der Hagel noch die Niederschlagsmenge von $28,7 \mathrm{l} / \mathrm{m}^{2}$ zeigten Auswirkungen auf den Lachnidenbesatz.

Der leichte Rückgang im Juli und August erfolgte kontinuierlich; er wurde weder während der Trockenperiode der 2. Julihälfte verzögert noch wurde er durch die häufigen Niederschläge von Ende Juli bis Anfang September beschleunigt.

1980 (Abb. 5 und 6) : Der Massenwechsel 1980 ähnelte dem von 1978. Der im Mai festgestellte Besatz an erwachsenen Stammüttern betrug durchschnittlich 1 Tier je Baum. Die ersten $F_{1}$ wurden am 21.5. gezählt. Im Mittel der 6 Bäume stieg die Befallsdichte bis zum 26.6. kontinuierlich auf den höchsten Wert von durchschnittlich 92 Tieren je Baum an. Im Juli erfolgte ein rapider Rückgang im Lachnidenbesatz, der sich im August/September etwas abflachte.

Die Witterung der Vermehrungsphase 1980 lässt sich in 3 Perioden einteilen : regnerisch und kühl war es vom 27.5. bis 3. Juni; überwiegend zu warm war es vom 4 . bis zum 17. Juni; nach der vom 4. bis 13.6. währenden niederschlagsarmen Periode regnete es täglich ab 14.6. bis Monatsende mit unterschiedlicher Intensität, dabei war es erheblich zu kühl und sonnenscheinarm. Dieser extreme Witterungscharakter blieb bis zum 22.7. erhalten.

Der im Durchschnitt von 6 Bäumen gemessene, steile und kontinuierliche Anstieg der Populationsdichte lässt den Schluss zu, dass die Vermehrungstätigkeit weder durch die warm-trockene Witterung begünstigt noch durch die kalt-feuchte Witterung gehemmt worden ist. Deutlich wird dies auch dann, wenn man den Massenwechsel auf einzelnen Bäumen miteinander vergleicht (Abb. 6). 


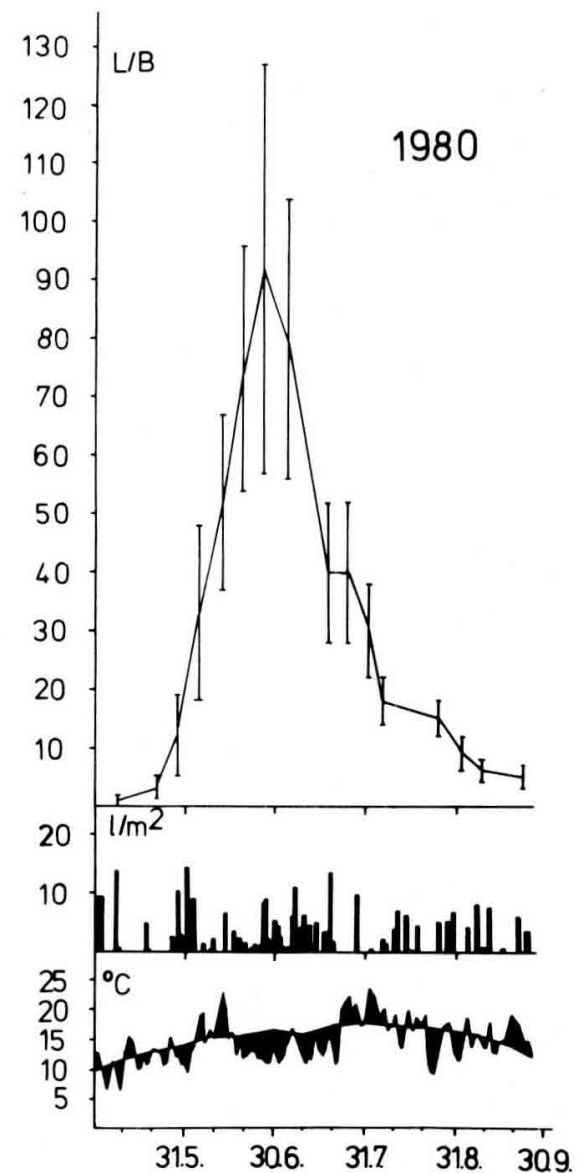

Aвв. 5. - Der Massenwechsel von C. pectinatae und der Verlauf der Witterung 1980 von Mai bis September.

Populationsdichte, Niederschlagsmenge und Temperatur wie in Abb. 1.

Fig. 5. - Population trend of C. pectinatae with temperature and rainfall data in 1980 (May to September).

Population density, rainfall data and temperature as in Fig. 1.

Auf Baum 4 stieg der Besatz innerhalb von 3 Wochen (vom 21.5. bis zum 12.6) von 1 auf 54 Tiere an, so dass auf diesem Baum schon am 12.6. der maximale Wert gemessen wurde. Anschliessend ging der Besatz auf Baum 4 langsam zurück, nach 6 Wochen wurden am 25.7. noch 40 Tiere gezählt; danach reduzierte sich der Besatz innerhalb von 2 Wochen um $50 \%$.

Auf Baum 3 dauerte die Vermehrungsphase 14 Tage länger als auf Baum 4. Am 26.6. wurden mit 151 Tieren dreimal so viel gezählt wie auf Baum 4. Während auf Baum 4 der Lachnidenbesatz nur langsam abnahm, erfolgte auf Baum 3 innerhalb von 3 Wochen ein Rückgang um 109 Tiere auf 42. 


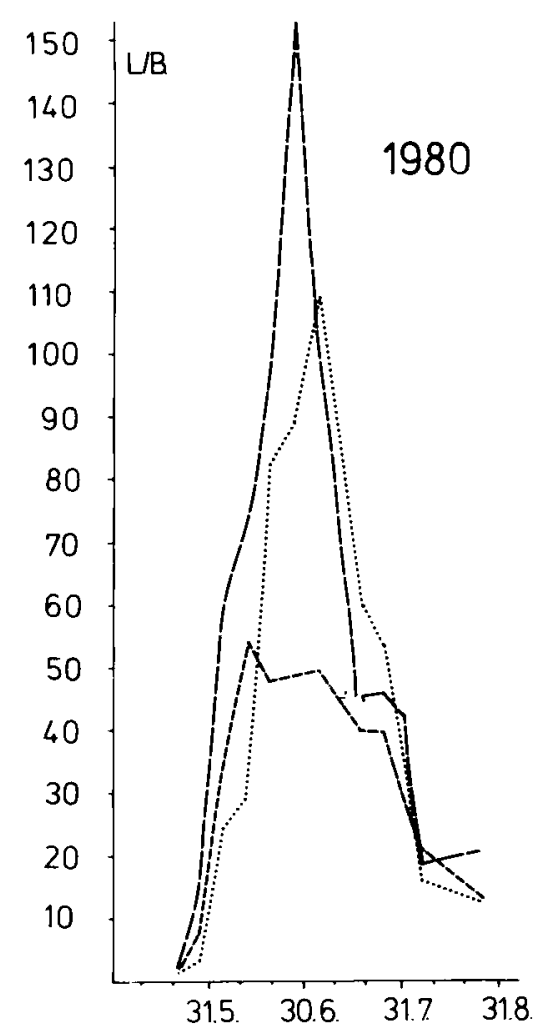

Aв8. 6. - Der Massenwechsel von C. pectinatae 1980 von Mai bis August auf Baum 2 (…….....),

Baum 3 (_-__- ) und Baum 4 (_-

FIG. 6. - Population trend of C. pectinatae in 1980 (May to August) on tree $2(\ldots . . . . . . . .$.$) , tree 3(---C-)$ and tree $4(------)$.

Auf Baum 2 wurde das Befallsmaximum von 109 Tieren erst am 4.7. festgestellt.

Das Ende der Vermehrungsphase und der Beginn des Zusammenbruchs fielen 1980 in eine Periode feucht-kalter Witterung, die vom 14.6. bis zum 22.7. andauerte. Der Zusammenbruch wurde in der Zeit zwischen dem 18.7. und dem 23.7. kurz gestoppt, an beiden Tagen wurden im Durchschnitt 40 Tiere je Baum gezählt. In dieser Zeit wurde die feucht-kalte Witterung am 23.7. durch eine warme und trockene Periode abgelöst, die bis zum 10.8. andauerte.

Zwischen dem 18.7. und dem 25.7. fiel lediglich am 20.7. mit $14,0 \mathrm{l} / \mathrm{m}^{2}$ bemerkenswerter Niederschlag.

Vom 25.7. bis zum 7.8. ging die Populationsdichte von 40 auf 18 Tiere zurück, obwohl in dieser Zeit die Witterung warm und trocken blieb. Starke Niederschläge fielen nur während eines Gewitters am 29.7. mit $9,71 / \mathrm{m}^{2}$. 
1981 (Abb. 7) : 1981 wurden im Mai wie 1979 und 1980 im Durchschnitt 1 Stammutter je Baum gezählt. Die ersten $F_{1}$ wurden am 28.4. während eines vom 16.4. bis 6.5. herrschenden Kälteeinbruchs festgestellt. Die Zahl der Tiere nahm erst deutlich $\mathrm{zu}$, als am 7.5. bis zum 10.5. übernormale Temperaturen gemessen wurden. Der Lachnidenbesatz stieg von durchschnittlich 2 Tieren je Baum am 8.5. auf 16 Tiere am 29.5. an, blieb anschliessend bis zum 5.6. nahezu konstant, um dann innerhalb einer Woche auf den maximalen Wert von 27 Tieren je Baum (am 11.6.) anzuwachsen.

Die Witterung während der Vermehrungsphase war gekennzeichnet durch starke Temperaturschwankungen. Bis zum 6.5. war es zu kalt, zwischen dem 7.5. und dem 23.5. war es insgesamt zu warm. Die sich anschliessende kräftige Abkühlung wurde am 30.5. von sehr warmer Witterung abgelöst. Mit Ausnahme des 4.6. wurden in der ersten Junihälfte jeden Tag überdurchschnittliche Temperaturen gemessen. Die Kälteperiode Ende Mai hatte den Populationsanstieg offensichtlicn nicht beeinflusst, dagegen verzögerte sich der Anstieg während der hochsommerlichen Tage Anfang Juni.

Die z.T. heftigen Niederschläge am 23., 24., 25. und 26.5. zeigten keinen negativen Einfluss; genausowenig wurde ein positiver Einfluss der warmen und trockenen Witterung am 31.5., 1. und 2.6. festgestellt, der Besatz ging während dieser Tage sogar leicht zurück. Bei Gewitter wurden am 3.6. $20,71 / \mathrm{m}^{2}$ gemessen, während rund 10 Minuten fiel Hagel. Bei der Zählung am 5.6. wurde im Durchschnitt mit 17 Tieren je Baum 1 Lachnide mehr gezählt als am 1. Juni.

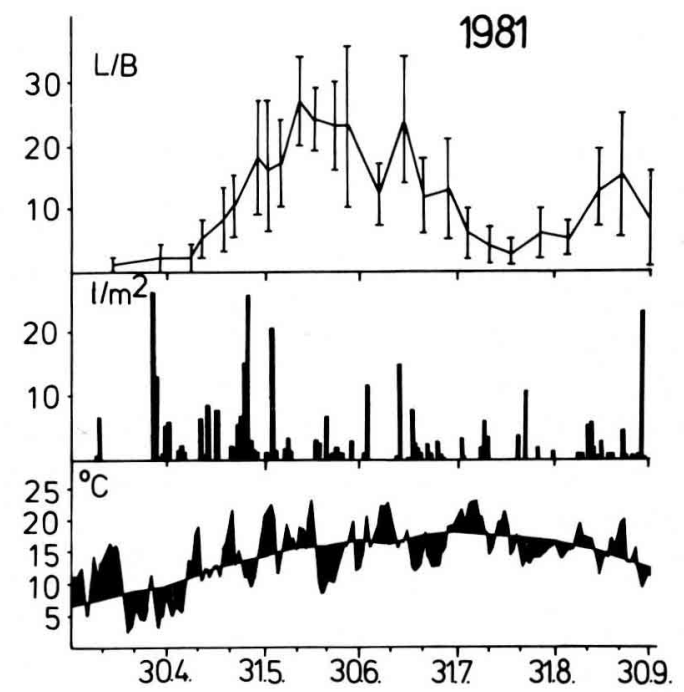

Aвв. 7. - Der Massenwechsel von C. pectinatae und der Verlauf der Witterung 1981 von April bis September.

Populationsdichte, Niederschlagsmenge und Temperatur wie in Abb 1.

FIG. 7. - Population trend of $\mathrm{C}$. pectinatae with temperature and rainfall data 1981 (April to September).

Population density, rainfall data and temperature as in Fig. 1. 
Vom 5.6. bis zum 16.6. war es trocken, nur an 3 Tagen fiel geringfügiger Niederschlag. In dieser Periode ging die Vermehrungsphase zu Ende, vom 11.6. bis zum 26.6. blieb die Populationsdichte nahezu konstant, sie nahm dann innerhalb einer Woche von 23 Tieren auf 12 je Baum ab, um anschliessend wieder auf 24 Tiere (14.7.) anzusteigen. Ein Einfluss der Witterung auf diese Veränderungen in der Populationsdichte ist nicht erkennbar.

Nach dem 14.7. setzte der Zusammenbruch ein. Am 17.8. wurde ein Minimum von 3 Lachniden je Baum gemessen.

Während der ersten Hälfte des Zusammenbruchs war die Witterung insgesamt zu kalt und niederschlagsarm, wenn auch vom 14.7. bis zum 28.7. 10 Regentage gezählt wurden, wobei die festgestellten Niederschlagsmengen nur um $31 / \mathrm{m}^{2}$ lagen und lediglich am 13.7. 15,0 1/ $\mathrm{m}^{2}$ gemessen wurden.

Während der zweiten Hälfte des Zusammenbruchs herrschte hochsommerliches Wetter mit nur 4 Regentagen; gebremst wurde der Rückgang durch diese Witterung nicht.

Im September stieg die Populationsdichte - wie 1977 - bei einer insgesamt warmen und trockenen Witterung erneut an. Am 21.9. wurden im Durchschnitt 17 Geschlechtstiere je Baum gezählt. Der Besatz ging bis zum 30.9. etwa um die Hälfte zurück. Am 30.9. wurde das erste Winterei gefunden.

\section{DISKUSSION}

Die auffälligsten Unterschiede im Massenwechsel zwischen den Jahren betreffen den Schlüpftermin der Stammütter, den Zeitpunkt des Auftretens der ersten $F_{1}$, die Dauer der Vermehrungsphase sowie den Zeitpunkt und die Höhe des maximalen Befalls (Tab. 1 und 2). Der Schlüpftermin ist mit dem Monatsmittel der Temperatur von Februar und März korreliert (vgl. Tab. 3). So ist das zeitige Schlüpfen der Stammütter 1977 vor allem durch die sehr warme Witterung im Februar und März verursacht worden. 1978, 1979 und 1980 schlüpften die Stammütter etwa 3-4 Wochen später, 1981 aufgrund des warmen Märzes nur etwa 2 Wochen später.

Der Temperaturverlauf im März und April beeinflusst weiterhin die Entwicklungsgeschwindigkeit der Stammütter und damit den Zeitpunkt des Auftretens der ersten $F_{1} .1978$ und 1981 war der April deutlich wärmer als 1979 und 1980, entsprechend früher traten die ersten $F_{1}$ auf (Anfang Mai bzw. Ende April). 1977 wurden die ersten $F_{1}$ ebenfalls schon Anfang Mai gezählt, obwohl der April mit $6,4{ }^{\circ} \mathrm{C}$ relativ kalt war. Wahrscheinlich hatte der warme März 1977 die Entwicklung der Stammütter derart beschleunigt, dass ihre Vermehrungstätigkeit trotz des Kälterückschlages im April frühzeitig einsetzte. 
TAB. 2. - Dichte-Daten des Massenwechsels von C. pectinatae.

TABL. 2. - Population density of C. pectinatae in May, June and August, 1977-1981.

\begin{tabular}{|c|c|c|c|c|c|}
\hline & 1977 & 1978 & 1979 & 1980 & 1981 \\
\hline $\begin{array}{l}\text { Durchschnittlicher Besatz an erwachsenen Stam- } \\
\text { müttern je Baum (Anfang Mai) }\end{array}$ & \multirow[t]{2}{*}{3} & \multirow[t]{2}{*}{2} & \multirow[t]{2}{*}{1} & \multirow[t]{2}{*}{1} & \multirow[t]{2}{*}{1} \\
\hline $\begin{array}{l}\text { Mean population of adult fundatrices on each tree (early } \\
\text { May) }\end{array}$ & & & & & \\
\hline $\begin{array}{l}\text { Durchschnittlicher maximaler Besatz je Baum (im } \\
\text { Juni) }\end{array}$ & \multirow[t]{2}{*}{41} & \multirow[t]{2}{*}{84} & \multirow[t]{2}{*}{29} & \multirow[t]{2}{*}{92} & \multirow[t]{2}{*}{27} \\
\hline Means of maximum population on each tree (in June) & & & & & \\
\hline Durchschnittlicher Besatz je Baum (im August) & 6 & 8 & 7 & 7 & 5 \\
\hline Mean population on each tree (in August) & & & & & \\
\hline
\end{tabular}

LEONHARDT (1940) untersuchte die Entwicklungsdauer der einzelnen Generationen von C. pectinatae, indem er Lachnidenzuchten auf abgeschnittenen, in Nährlösung gestellten Tannenzweigen unter verschiedenen Temperaturbedingungen hielt. Am kürzesten war die Entwicklungszeit aller Generationen in der Zucht, die im Zimmer bei gleichmässig hohen Temperaturen $\left(15-20^{\circ} \mathrm{C}\right)$ gehalten wurde. Länger war die Entwicklung unter natürlichen klimatischen Aussenbedingungen, in der $F_{1}-$ und $\mathrm{F}_{2}$ - Generation betrug sie etwa 40 Tage, im Zimmer dagegen nur durchschnittlich 21 Tage. Allerdings war im Versuch nicht zu erkennen, dass eine kürzere Entwicklungsdauer die Fruchtbarkeit erhöht (vgl. LeonHARDT, 1940, Tab. 2).

Die Beobachturg von C. pectinatae in Hohenheim führte zu einem ähnlichen Ergebnis. Es besteht keine Korrelation zwischen dem Schlüpftermin und dem Zeitpunkt des Auftretens der ersten $F_{1}$ einerseits und der Vermehrungsrate und der Dauer der Vermehrungsphase anderseits. Die Höhe der Vermehrungsrate und die Dauer der Vermehrungsphase können nur - wenn überhaupt mit der Witterung - mit einem indirekten Einfluss der Witterung über den Wirtsbaum auf die Lachniden erklärt werden. Ein direkter Einfluss der Witterung auf den Massenwechsel während der Vermehrungsphase und während des Zusammenbruchs ist nicht vorhanden. Diese Aussage wird mit folgenden Ergebnissen begründet :

1. Bei dem direkten Vergleich des Witterungsverlaufs mit dem Massenwechsel von 1977 bis 1981 ist nicht erkennbar, dass die Lachnidenentwicklung während der Vermehrungsphase bei ungünstigen Witterungsbedingungen gehemmt bzw. bei günstigen Bedingungen gefördet wird. Genausowenig zeigt sich ein (direkter) Einfluss der Witterung auf den Massenwechsel während des und nach dem Zusammenbruch.

2. Auf benachbart stehenden Bäumen traten z.T. sehr grosse Unterschiede im Massenwechsel auf, obwohl diese Bäume den gleichen Witterungsbedingungen ausgesetzt waren.

3. 1978 und 1980 vermehrte sich C. pectinatae stärker, obwohl Mai und Juni kälter und 1978 auch niederschlagsreicher waren als 1977, 1979 und 1981. 


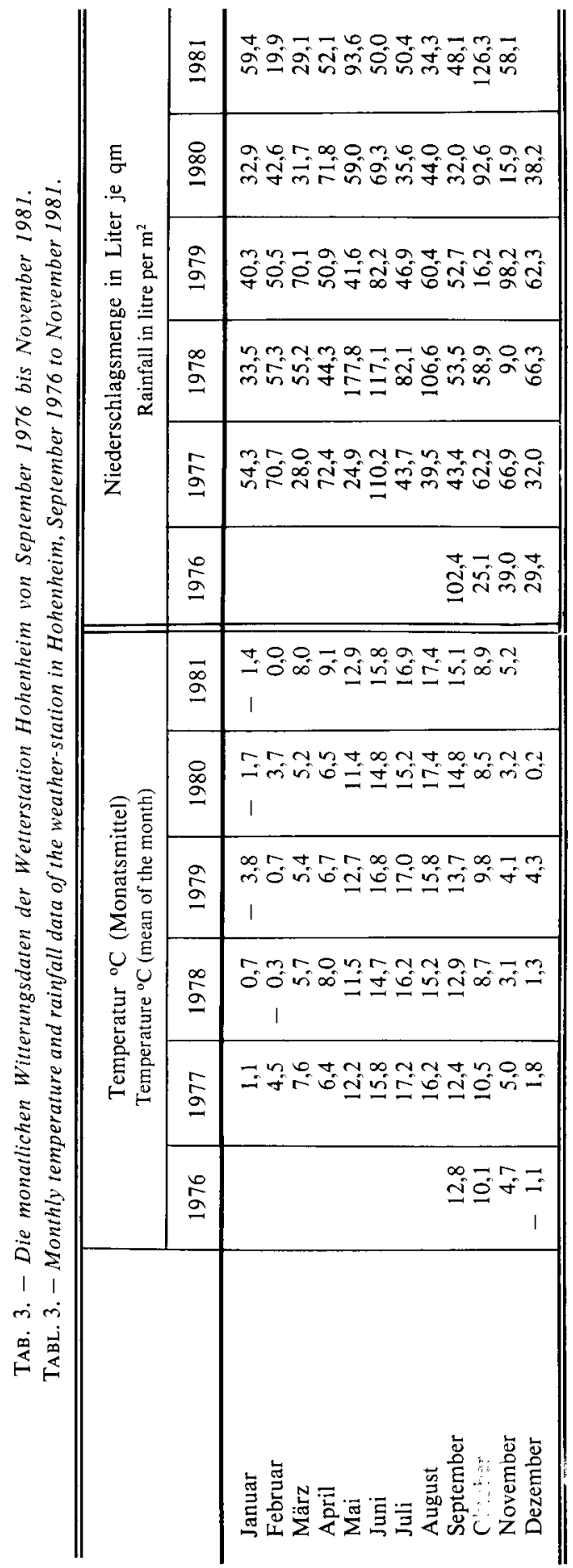


Diese Erkenntnis bedeutet gleichzeitig, dass das von Jahr zu Jahr unterschiedlich starke Auftreten von C. pectinatae nicht mit dem Einfluss der Witterung erklärt werden kann. Das Ausbleiben eines Massenbefalls von Lachniden wurde wiederholt damit begründet, dass die Witterung im Mai und Juni - in den für die Entwicklung eines Massenbefalls entscheidenden Monaten - zu kalt oder zu niederschlagsreich war (z.B. WePFER, 1959; Singer, 1959, SCHMID, 1968). Allerdings wurde in einigen Fällen auch von einer Waldtracht im Juli/August berichtet, der ein kalter und regnerischer Juni vorausgegangen war (NEUENSCHWANDER, 1951; MONDL, 1975).

LEONHARDT (1940), Wille (1966, 1967), Wellenstein (1977) und WÜTHERICH (1977) haben versucht, den Einfluss der Witterung auf den Massenwechsel der Honigtauerzeuger dadurch zu klären, indem sie den Witterungsverlauf von Fehljahren mit dem von Honigjahren anhand der Abweichungen der Monatsmittel von den langjährigen Werten der Temperatur und des Niederschlags verglichen. Dabei prüfte allein WiLle den Zusammenhang zwischen der Witterung und dem Ertrag aus der Honigtautracht nicht an ausgesuchten Jahren, sondern über den gesamten Zeitraum von 1893 bis 1964; ihm standen dabei die Waagstockmessungen von 6 Beobachtungsstationen in der Schweiz zur Verfügung. WILLE fand im Gegensatz zu LEONHARDT, WELLENSTEIN und WüthERICH keinen Zusammenhang zwischen dem Witterungsverlauf und dem Honigertrag, wobei besonders überzeugt, dass er lückenlose Aufzeichnungen eines Zeitraumes von 72 Jahren verwenden konnte.

Der Honigertrag allein ist kein Masstab für den Massenwechsel der Honigtauerzeuger; er hängt nicht nur von der Befallsdichte der Honigtauerzeuger ab, sondern auch - wie eingangs schon erwähnt - von der Leistungsstärke der Bienenvölker, von den Flugbedingungen während einer Honigtautracht und von der Attraktivität des Honigtaus (besonders bei gleichzeitig auftretender Blütentracht).

Ausserdem wird auch nicht berücksichtigt, dass die verschiedenen Honigtauerzeuger sich im Entwicklungszyklus bzw. im Massenwechsel zum Teil erheblich unterscheiden. So kann eine Fichtentracht entweder von Lecanien oder von Lachniden stammen. Bei beiden ist die Entwicklung eines Massenbefalls erste Voraussetzung für eine Honigtautracht. Während bei den Lachniden eine Massenvermehrung im Mai/Juni zu einer Honigtautracht im Juni bzw. Juli des gleichen Jahres führt, entscheidet bei den Lecanien ihre Vermehrungsrate im Spätsommer des Vorjahres, ob es (im folgenden Jahr) zu einer Tracht kommt. Die witterungsempfindlichen Phasen der Lecanienentwicklung sind die Kopulation und besonders das Schlüpfen und Ausschwärmen der Lecanienlarven im Spätsommer des Vorjahres. Insgesamt scheint auch bei den Lecanien der Einfluss der Witterung auf den Massenwechsel nicht wesentlich zu sein (PECHHA KER, 1977).

Die wichtigsten Honigtau erzeugenden Lachnidenarten weisen ebenfalls verschiedene Eigentümlichkeiten auf, die hei der keurteilung der Witterung auf die Entstehung einer Waldtracht berücksichtig̨ werden müssen. So lebt C. pectinatae im Gegensatz zu anderen Lachnidenarten nicht in Kclonien, auch bei Massenbefall bleibt 
sie Einzelsitzer. Ausserdem liegt bei C. pectinatae der Anteil der Geflügelten an der Gesamtpopulation im Frühjahr und im Sommer stets unter $5 \%$ (MAQuELIN, 1974).

Die imkerlich wichtigsten Lachniden auf der Fichte sind C. pilicornis und C. piceae. Beide Arten bilden bei Massenbefall Kolonien mit einem hohen Anteil geflügelter Tiere. C. pilicornis besiedelt bevorzugt die Maitriebe und ist dort den Witterungseinflüssen ausgesetzt. Allerdings scheinen heftige Niederschläge nur dann dezimierend zu wirken, wenn sich Lachniden besonders bei Übervölkerung ungeschützt auf der Oberseite der Triebe ansiedeln (vgl. ECKLOFF, 1972).

C. piceae ist im Juli/August auf Jungfichten am Stamm oder auf Altfichten an der nadellosen Unterseite stärkerer Äste zu finden. An diesen Saugorten ist C. piceae anders als $C$. pilicornis vor Niederschlägen geschützt, der am Stamm ausgeschiedene Honigtau wird auch bei längeren Regenfällen nicht abgewaschen und kann (wie wir 1980 beobachtet haben), sobald der Regen aufhört, von Bienen beflogen werden.

Diese Unterschiede in der Lebensweise verbieten es einerseits, aufgrund des Honigertrages Rückschlüsse auf den Massenwechsel der Honigtauerzeuger zu ziehen, anderseits aber auch, die bei der Beobachtung des Massenwechsels von C. pectinatae gewonnenen Erkenntnisse zu verallgemeinern.

Charakteristisch für den Massenwechsel von $C$. pectinatae sind die Vermehrungsphase und der Zusammenbruch. In Hohenheim war die Vermehrungsphase jedes Jahr auf die Zeit des Austriebes im Mai und Juni begrenzt. Der Zusammenbruch wurde regelmässig im Juli/August beobachtet. Die Massenwechselkurven zeigten 1978, 1979 und 1980 nur einen deutlichen Gipfel im Juni; 1977 und 1981 wurde neben diesem Maximum ein zweites in September festgestellt, das aber niedriger lag als im Juni.

Bei den bisher durchgeführten Untersuchungen über den Massenwechsel von $C$. pectinatae (Maquelin, 1974; Liebig, 1979 a; Liebig und SchlipF, 1981) wurde in der Regel nur ein deutliches Maximum festgestellt, das meistens im Juni, Juli oder August auftrat.

In einzelnen Fällen wurde aber auch eine Verschiebung der Vermehrungsphase in den Zeitraum Juli/August beobachtet, so dass der stärkste Befall mit dem Auftreten der Geschlechtstiere im September gemessen wurde.

Bei den Fichtenlachniden wurde in der Regel ebenfalls nur ein Populationsmaximum festgestellt (z.B. ThALENHORsT, 1972; ECKLOFF, 1972), lediglich SCHEURER (1964) hatte im September ein zweites neben dem im Juni beobachtet.

Der Massenwechsel von Blattläusen wird allgemein mit Schwankungen im Gehalt des Phloemsaftes an löslichen Stickstoffverbindungen erklärt (KLofT et al., 1965). Der Gehalt an Aminosäuren, Amiden, Vitaminen und anderen lebenswichtigen Stoffen im Phloemsaft ist bei Blattgehölzen während des Austriebes und vor dem Blattfall besonders hoch. Entsprechend sei ein Populationsmaximum nach Beendigung des Austriebes und ein weiteres vor Beginn des Blattfalls möglich. Das bei auf Koniferen 
lebenden Lachniden (manchmal) auftretende Populationsmaximum im Herbst führt ScHEURER (1964) darauf zurück, dass mit der Nährstoffeinlagerung im Spätsommer der Stickstoffgehalt des Phloemsaftes ansteigt. Allerdings wurde in vielen Fällen im Herbst keine Vermehrung der auf Koniferen lebenden Lachniden beobachtet, so dass $\mathrm{zu}$ vermuten ist, dass noch andere, bisher unbekannte Faktoren eine Rolle spielen.

Räuber, Parasiten und Krankheiten haben nach MAQueLIN (1974) und nach eigenen Beobachtungen wie die Witterung keinen wesentlichen Einfluss auf den Massenwechsel von C. pectinatae. Das Zustandekommen eines Massenbefalls bzw. sein Ausbleiben wird wahrscheinlich in erster Linie durch den physiologischen Zustand des Wirtsbaumes geregelt, auf den sowohl Witterungs- als auch Standortfaktoren wirken. 1981 blieb an vielen, sonst sicheren Tannentrachtstandorten der Massenbefall von $C$. pectinatae aus; nur an wenigen Plätzen im Raum Alpirsbach entwickelte sich ein starker Lachnidenbesatz, der für eine gute bis sehr gute Tracht ausreichte (KöGLER, 1981; LIEBIG und SCHLIPF, 1982).

Daraus und aus den Ergebnissen dieser Arbeit schlussfolgernd haben wir die Hypothese aufgestellt, dass die Unterschiede im Massenwechsel zwischen den Jahren bzw. zwischen den Standorten auf eine witterungs- und standortbedingte unterschiedliche Nährstoffversorgung der Weisstannen zurückzuführen sind.

Die Untersuchungen werden fortgesetzt.

DANK

Die Untersuchungen wurden von der Deutschen Forschungsgemeinschaft finanziell unterstützt.

Eingegangen im April 1982.

Reçu pour publication en avril 1982.

\section{SUMMARY}

WEATHER CONDITIONS AND POPULATION DYNAMICS

OF CINARA PECTINATAE (NÖRDL.) (HOMOPTERA, LACHNIDAE) 1977-1981.

In Hohenheim population dynamics of $C$. pectinatae feeding on $A$ bies alba were compared with meteorological conditions in the years 1977-1981. Population denstiy was measured on trees 15-20 years old. Lachnids were counted weekly or fortnightly at marked branches (circa $20 \times 30 \mathrm{~cm}, 10$ branches each tree). The development of weather was described with the daily and monthly mean temperature and rainfall.

Population dynamics of $C$. pectinatae run off in the same way every year. For that reason a model of population dynamics was formulated, which was classified in 4 or 5 phases from March till October :

1. The time of growth and development of the fundatrices (March/April/May).

2. The phase of reproduction (May/June).

3. Stationary phase of population density (June).

4. (or 3.). Decrease of population density (July/August).

5. (or 4.). Appearance and development of sexuales (September/October). 
As a rule, reproduction of $C$. pectinatae is numerous only during sprouting of trees in May and June. In 1978 and 1980 , during the summer a greater number of $C$. pectinatae was measured than in 1977, 1979 and 1981 . In 1978 and 1980 reproduction was better, although the weather in May and June was colder. Moreover, in 1978 there was more rainfall in May and June than in the other years.

The comparison of weather conditions with population dynamics shows that variations in population density is not caused by rainfall and by changes of temperature.

Fertility and mortality of $C$. pectinatae are influenced especially by the physiological predisposition of the host-plant. The investigations will be continued with the hypothesis, that the differences of population dynamics between the years or between locations are the result of a variable supply of the firs with nutrients dependent on weather and location conditions.

\section{RESUME \\ CONDITIONS MÉTEOROLOGIQUES ET DYNAMIQUE DES POPULATIONS \\ DE CINARA PECTINATAE (HOMOPTERA, LACHNIDAE) de 1977 à 1981}

On a comparé à Hohenheim la dynamique des populations de Cinara pectinatae sur le sapin Abies alba avec les conditions météorologiques durant la période 1977-1981. La densité de population a été mesurée sur des arbres âgés de 15 à 20 ans. Toutes les semaines ou tous les quinze jours on a dénombré les lachnides sur des branches marquées (environ $20 \times 30 \mathrm{~cm}, 10$ branches par arbre). Les conditions météorologiques ont été suivies par les températures moyennes et la pluviométrie journalières et mensuelles

La dynamique des populations de $C$. pectinatae suit chaque année le même modèle, que l'on peut découper en 4 à 5 phases de mars à octobre :

1. Phase de croissance et de développement des fondatrices (mars à mai).

2. Phase de reproduction (mai à juin).

3. Phase de stagnation de la densité de population (juin).

4 (ou 3). Chute de la densité de population (juillet-août).

5 (ou 4). Apparition et développement des sexués (septembre-octobre).

La multiplication des lachnides n'a lieu en général que pendant la poussée des arbres en mai et juin. En juin 1978 et 1980 on a dénombré 2 à 3 fois plus de lachnides qu'en 1977, 1979 et 1981 . Ces deux années là $C$. pectinatae s'est plus reproduit, bien que le temps ait été plus froid et plus pluvieux. La comparaison de la dynamique des populations avec les conditions météorologiques montre que les variations de la densité de population ne sont pas dues aux précipitations ni aux fluctuations de température.

La fęrtilité et la mortalité des lachnides sont vraisemblablement influencées principalement par l'état physiologique de l'arbre-hôte. Les recherches se poursuivent avec l'hypothèse que les différences de dynamique des populations d'une année à l'autre et d'un endroit à un autre sont à attribuer à une variation dans l'approvisionnement des sapins en éléments nutritifs, variation liée aux conditions météorologiques et géographiques.

\section{LITERATUR VERZEICHNIS}

ECKLOFF W., 1972. - Beitrag zur Ökologie und forstlichen Bedeutung bienenwirtschaftlich wichtiger Rindenläuse. Z, ang. Ent., 70, 134-157.

Kloft W., Maurizio A. und Kaeser, W., 1965. - Das Waldhonigbuch. Imkerfreund Bücher Bd. 3, München : Ehrenwirth. 
KöGLER F., 1981. - Beobachtungsbericht für Juli 1981 bzw. für August 1981. Allg. Dt. Imkerztg., 281 und 315 .

LeONHARdT H., 1940. - Beiträge zur Kenntnis der Lachniden, der wichtigsten Tannenhonigtauerzeuger. Z. ang. Ent. 27, 208-272.

Liebig G., 1979 a. - Eine Analyse der Tannentracht 1977 und 1978. Allg. Dt. Imkerztg., 1-5.

Liebig G., 1979 b. - Hinweise für die Beobachtung der Tannentracht. Allg. Dt. Imkerztg., 65-67, 101 103, 129-131, 204-205, 337-338.

Liebig G. und SchlipF U., 1981. - Ergebnisse der Waldtrachtforschung. Allg. Dt. Imkerztg., 165-167.

Liebig G. und SCHLIPF U., 1982. - Die Tannentracht 1981 : Vorhersage und Verlauf. Allg. Dt. Imkerztg., (7) 201-204.

MaQuelin Ch., 1974. - Observations sur la biologie et l'écologie d'un puceron utile à l'apiculture : Buchneria pectinatae (Nördl.) (Homoptera, Lachnidae). Dissertation, Zürich.

MondL J., 1975. - Waldtracht 1974. Bienenvater, 55.

NeUENSChWANder H., 1951. - Waldtracht abhängig von Frost? Schw. Bienenztg., 129-130.

PechHacker H., 1977. - Uber die Auswirkung von Umwelteinflüssen auf die Populations-Entwicklung der Physokermes-Arten. Apidologie, 8, 451-457.

SCHEURER S., 1964. - Generationenfolge und Aufenthaltsorte der im östlichen Teil des Harzes gefundenen Lachniden. Dt. Bienenwirtschaft, 15, 214-217.

SCHMiD S., 1968. - Die Entwicklung der Lachniden auf der Tanne 1967. Bienenvater, 157-158 und 188.

SChwerdffeger F., 1944. - Die Waldkrankheiten. Berlin : Paul Parey.

SingER W., 1959. - Weisstannentrachtbeobachtungen. Bienenvater, 223.

Wellenstein G., 1977. - Die Grundlagen der Waldtracht und Möglichkeiten ihrer bienenwirtschaftlichen Nutzung. Z. ang. Zool. 64, 291-309.

WEPFER T., 1959. - Weisstannentracht und Niederschläge Schw. Bienenztg., 7-14.

Wille H., 1966. - Verlauf der Juni-Trachten in den Jahren 1893-1964. Schw. Bienenztg., 13-24.

WiLle H., 1967. - Verlauf der Juli-Trachten in den letzten siebzig Jahren und Vergleich mit den entsprechenden Juni-Trachten. Schw. Bienenztg., 229-236 und 290-297.

WÜTHERICH G., 1977. - Untersuchungen über Honigtau, insbesondere über die Witterungsabhängigkeit des Massenwechsels Honigtau erzeugender Lachniden und die daraus resultierenden guten und schlechten Honigjahre. Diplomarbeit, Freiburg.

Thalenhorst W., 1972. - Düngung, Wuchsmerkmale der Fichte und Arthropodenbefall. Mitt. Nieders. Landesforstverwaltung, 18. 